\title{
Protocolos de Estresse Cardiovasculares
}

Adição de exercício às provas de estímulo farmacológico - A associação de exercício dinâmico em baixa carga de trabalho total, como caminhar em esteira rolante até o máximo de dois estágios do protocolo de Bruce, tem evidenciado a redução da atividade subdiafragmática (hepática) e melhora na relação da radioatividade órgão-alvo/ vísceras (background), com conseqüente melhora da qualidade das imagens ${ }^{1,2}$. Da mesma forma, observa-se diminuição da ocorrência e intensidade dos efeitos colaterais decorrentes da infusão de dipiridamol ou adenosina, bem como da incidência de bloqueios atrioventriculares ${ }^{1,2}$.

Novos Fármacos ${ }^{3}$ - A utilização de antagonistas específicos dos receptores $A_{1}$ (de denominação N-0861) é promissora para a redução de efeitos adversos sem comprometer a eficácia da adenosina em relação à vasodilatação coronariana. Da mesma maneira, agonistas seletivos dos receptores $A_{2}$ (GGS-21680, MRE-0470, CVT-3146, ATL-193, ATL-146e), ainda sob estudo, têm evidenciado hiperemia coronariana adequada e menor intensidade de efeitos sistêmicos, especialmente dor torácica e bloqueio atrioventricular. Adicionalmente, se efetivamente não produzirem broncoespamo como esperado, poderão ter sua aplicação ampliada de modo importante. Udelson e cols ${ }^{4}$, comparando o estresse farmacológico com binodenoson (agonista $A_{2}$ ) e com adenosina, verificaram capacidade similar de demonstração da gravidade e extensão da isquemia e da relação dose-dependente dos efeitos adversos.

Arbutamina - A arbutamina é uma catecolamina sintética desenvolvida para ser usada como agente estressante farmacológico que, recentemente, teve sua utilização, na cintilografia de perfusão miocárdica e na ecocardiografia, aprovada pelo FDA. Este fármaco é um agonista misto ${ }^{5}$ beta- 1 e beta- 2 , com uma moderada afinidade por receptores alfa-1. A arbutamina possui uma resposta cronotrópica semelhante à da dobutamina, porém com menor efeito vasodilatador periférico. É utilizada em um sistema computadorizado de infusão contínua, que monitora, constantemente, a resposta da freqüência cardíaca durante a administração venosa do fármaco. 0 conjunto consiste em um computador, um monitor de canal único de ECG, um monitor de PA não invasiva e um acesso venoso. O médico seleciona o incremento apropriado da freqüência cardíaca para cada paciente. 0 sistema calcula a dose e regula o fluxo de administração para que a freqüência cardíaca preconizada seja atingida ${ }^{5}$. Os efeitos adversos mais freqüentes são: tremores $(22 \%)$, tontura (11\%), cefaléia (11\%), parestesia $(7 \%)$, arritmias $(6 \%)$ e hipotensão $(4 \%)^{6}$.

\section{REFERÊNCIAS}

1. Elliot MD, Holly TA, Cook $C$ et al. The impact of an abbreviated adenosine incorporating adjunctive treadmill exercise on side effects and image quality in patients undergoing stress myocardial perfusion imaging. J Nucl Cardiol 2000; 7: 584-89.

2. Vítola JV, Brambatti JC, Caligaris F et al. Exercise supplementation to dipyridamole prevents hypotension, improves electrocardiogram sensitivity and increases heart-to-liver ratio on Tc-99m Sestamibi imaging. J Nucl Cardiol 2001; 8:652-59.

3. Udelson JE, Heller GV, Wackers FJ et al. Randomized controlled doseranging study of the selective adenosine $\mathrm{A} 2 \mathrm{~A}$ receptor agonist binodenoson for pharmacological stress as an adjunct to myocardial perfusion imaging. Circulation 2004;109: 457-64.

4. Hendel RC. New developments in pharmacologic stress myocardial perfusion imaging. $7^{\text {th }}$ Annual Symposium and Scientific Session. ASNC. Syllabus 2002; 47-50.

5. Marwick TH. Arbutamine stress testing with closed loop drug delivery: toward the ideal or just another pharmacologic stress technique? J Am Coll Cardiol 1995; 26: 1176-79.

6. Raza J A, Reeves WC, Movahed A. Pharmacological stress agents for evaluation of ischemic heart disease. Inter J Cardiol 2001; 8:157-67.

7. Cohen JL, Chan KL, Jaarsma W et al., Arbutamine echocardiography: efficacy and safety of a new pharmacologic stress agent to induce myocardial ischemia and detect coronary artery disease. The International Arbutamine Study Group. J Am Coll Cardiol 1995; 26: pp. 1168-1175. 